ARTICLE

Received 27 Dec 2012 | Accepted 26 Jun 2013 | Published 12 Aug $2013 \quad$ DOl: 10.1038/ncomms3191

\title{
Root-derived CLE glycopeptides control nodulation by direct binding to HAR1 receptor kinase
}

Satoru Okamoto ${ }^{1,2}$, Hidefumi Shinohara ${ }^{1}$, Tomoko Mori ${ }^{1}$, Yoshikatsu Matsubayashi ${ }^{1} \&$ Masayoshi Kawaguchi ${ }^{1}$

Leguminous plants establish a symbiosis with rhizobia to enable nitrogen fixation in root nodules under the control of the presumed root-to-shoot-to-root negative feedback called autoregulation of nodulation. In Lotus japonicus, autoregulation is mediated by CLE-RS genes that are specifically expressed in the root, and the receptor kinase HAR1 that functions in the shoot. However, the mature functional structures of CLE-RS gene products and the molecular nature of CLE-RS/HAR1 signalling governed by these spatially distant components remain elusive. Here we show that CLE-RS2 is a post-translationally arabinosylated glycopeptide derived from the CLE domain. Chemically synthesized CLE-RS glycopeptides cause significant suppression of nodulation and directly bind to HAR1 in an arabinose-chain and sequencedependent manner. In addition, CLE-RS2 glycopeptide specifically produced in the root is found in xylem sap collected from the shoot. We propose that CLE-RS glycopeptides are the long sought mobile signals responsible for the initial step of autoregulation of nodulation.

\footnotetext{
${ }^{1}$ National Institute for Basic Biology, Nishigonaka 38, Myodaiji, Okazaki 444-8585, Japan. ${ }^{2}$ Research Fellow of the Japan Society for the Promotion of Science, 5-3-1 Kojimachi, Chiyoda-ku, Tokyo 102-0083, Japan. Correspondence and requests for materials should be addressed to Y.M.

(email: ymatsu@nibb.ac.jp) or to M.K. (masayosi@nibb.ac.jp).
} 
$\mathrm{M}$ any leguminous plant species establish a symbiotic association with rhizobia that induces the formation of nodules on roots. This symbiosis enables nitrogen fixation in the nodules and is beneficial to the host plants; however, excessive nodule formation is deleterious to the plants because the energy cost outweighs the need for fixed nitrogen. To achieve a balance in this symbiotic relationship, the host plant tightly controls the number of nodules via a root-to-shoot-to-root negative feedback signalling loop commonly termed autoregulation of nodulation ${ }^{1-4}$.

The initial step of autoregulation is thought to involve longdistance signalling from the root to the shoot ${ }^{5-7}$. This hypothesis was strongly supported by the findings from analyses of the Lotus japonicus hypernodulating mutant, hypernodulation aberrant root formation (har 1$)^{8-10}$, and orthologous mutants in other leguminous species $8,9,11,12$. HAR1 encodes a leucine-rich repeat receptor kinase (LRR-RK) that is expressed in both roots and shoots $^{8,9}$. Grafting experiments showed that HAR1 in the shoot is required to control nodule number in the root. Thus, HAR1 has been suggested to function in the shoot as a receptor for a signal emanating from the roots.

HAR1 has high similarity to Arabidopsis CLAVATA1 $(\mathrm{CLV} 1)^{13}$, a protein known to act as a receptor for the shoot apical meristem regulator CLV3 of the CLV3/ESR-related (CLE) small secreted peptide family ${ }^{14,15}$. A search for L. japonicus CLE family genes involved in nodulation identified two genes, CLE$R S 1$ and CLE-RS2 that are highly upregulated in roots that have been inoculated with rhizobia ${ }^{16}$. Overexpression of these CLE genes in hairy roots resulted in the systemic inhibition of nodulation in a HAR1-dependent manner. CLE-RS2 is also strongly upregulated in the roots of plants grown under high nitrate conditions that are known to abolish nodulation. Conversely, knockdown of Medicago truncatula MtCLE12 and $M t C L E 13$ resulted in a significant increase in nodule number ${ }^{17}$. Functionally similar CLE genes have also been identified in other leguminous species ${ }^{18-21}$. Thus, CLE-RS peptides are strong candidates for the root-derived signals that might act as ligands for HAR1. However, commercially synthesized CLE-RS peptides did not suppress nodulation ${ }^{16}$, suggesting that some specific modifications are required for the activity of CLE-RS peptides. Furthermore, the molecular basis of a CLE-RS/HAR1 signalling that involves such spatially distant components remains elusive.

In the present study, we used biochemical approaches to explore the bioactive forms of CLE-RS peptides and to identify their modes of action in long-distance regulation of nodulation. We found that arabinosylated CLE-RS peptides suppress nodulation and directly bind to HAR1 receptor kinase. Furthermore, we show that CLE-RS2 glycopeptide can travel from the roots to the shoot. These findings suggest that the CLERS peptides mediate organ-to-organ communication.

\section{Results}

CLE-RS2 is an arabinosylated glycopeptide. Secreted peptides involved in signalling pathways in plants often undergo posttranslational modification and proteolytic processing that alter their biological functions and specific receptor interactions ${ }^{22,23}$. To determine the mature functional structure of CLE-RS peptides, we used a whole-plant submerged-culture system to analyse secreted peptides that had accumulated in the apoplast of Arabidopsis plants overexpressing CLE-RS2. Under submergedculture conditions, any secreted peptides in the apoplast diffuse directly into the culture medium ${ }^{24}$. Nano-liquid chromatography mass spectrometry (nano-LC-MS) and nano-liquid chromatography tandem mass spectrometry (nano-LC-MS/MS) analyses of peptides that diffused from the apoplast identified a peak that eluted at $34.9 \mathrm{~min}\left(\mathrm{~m} / \mathrm{z} 908.9[\mathrm{M}+2 \mathrm{H}]^{2+}\right.$ and $\mathrm{m} / \mathrm{z} 606.3$ $\left.[\mathrm{M}+3 \mathrm{H}]^{3+}\right)$; this peak represents the mature 13 -amino-acid CLE-RS2 peptide that is derived from the C-terminal region of the precursor polypeptide (Fig. 1a-c). Notably, the seventh hydroxyproline $\left(\mathrm{Hyp}^{7}\right)$ residue of this peptide is posttranslationally modified with three residues of arabinose (Fig. 1d).

To determine whether the mature peptide structure of CLERS2 in L. japonicus is identical to that in Arabidopsis, we used Agrobacterium-mediated hairy root transformation to overexpress CLE-RS2 in L. japonicus; the secreted peptides that accumulated in the hairy root submerged-culture medium were analysed by nano-LC-MS and nano-LC-MS/MS. We detected a peak of $\mathrm{m} / \mathrm{z} 908.9$ and $\mathrm{m} / \mathrm{z} 606.3$ at $35.5 \mathrm{~min}$ that corresponds to the 13-amino-acid arabinosylated CLE-RS2 peptide (Fig. 1e). The MS/MS spectrum of this peak showed a fragmentation pattern identical to that of Arabidopsis, indicating that CLE-RS2 is also arabinosylated in L. japonicus (Supplementary Fig. S1). We concluded that the mature form of CLE-RS2 is a 13-amino-acid glycopeptide in which the $\mathrm{Hyp}^{7}$ residue is post-translationally modified with three residues of arabinose. Hereafter, we refer to this glycopeptide as $\left[\mathrm{Ara}_{3}\right] \mathrm{CLE}-\mathrm{RS} 2$.

Chemical synthesis of $\left[\mathrm{Ara}_{3}\right]$ CLE-RS glycopeptides. Modification of Hyp residues by arabinose is a plant-specific post-translational modification of peptides synthesized through secretory pathways ${ }^{25,26}$. A previous analysis of triarabinoside structures on the Arabidopsis CLV3 peptide showed that the Hyp residue was modified by three L-arabinose moieties with a linear $\beta-1,2$ linkage ${ }^{26}$. As $\left[\mathrm{Ara}_{3}\right]$ CLE-RS2 is present in plants at too low a level to obtain sufficient quantities for functional analyses, we decided to chemically synthesize $\left[\mathrm{Ara}_{3}\right] \mathrm{CLE}-\mathrm{RS} 2$. We based our synthesis on the method recently described for stereoselective synthesis of arabinosylated CLV3 in Arabidopsis ${ }^{27}$. In brief, a chemically synthesized triarabinosylated Hyp building block was incorporated into the peptide-resin at position $\mathrm{Hyp}^{7}$ using solid-phase peptide synthesis. After acidolytic release from the resin, the crude glycopeptide was deacetylated and purified by high-performance liquid chromatography (HPLC) to yield analytically pure $\left[\mathrm{Ara}_{3}\right] \mathrm{CLE}-\mathrm{RS} 2$. We confirmed that the retention time and MS/MS fragmentation pattern of the synthetic $\left[\mathrm{Ara}_{3}\right] \mathrm{CLE}-\mathrm{RS} 2$ were identical to those of natural [Ara 3 CLE-RS2 (Supplementary Fig. S2). We also prepared 13amino-acid $\left[\mathrm{Ara}_{3}\right]$ CLE-RS1 glycopeptide based on the sequence similarity of the conserved mature peptide domain.

[Ara ${ }_{3}$ ]CLE-RS glycopeptides suppress nodulation. To test whether $\left[\mathrm{Ara}_{3}\right] \mathrm{CLE}-\mathrm{RS} 2$ could act as a signal to negatively regulate nodulation, we applied various concentrations of synthetic [Ara 3 ]CLE-RS2 to L. japonicus seedlings. As HAR1 in the shoot is involved in the control of nodule number in the root, we applied the $\left[\mathrm{Ara}_{3}\right] \mathrm{CLE}-\mathrm{RS} 2$ solution directly to the shoot by cutting the surface of the cotyledons. We visually confirmed successful uptake by the appearance of a blue dye marker in the primary leaves of the shoot (Supplementary Fig. S3). In wild-type plants, concentrations of $\left[\mathrm{Ara}_{3}\right] \mathrm{CLE}-\mathrm{RS} 2$ as low as $100 \mathrm{nM}$ suppressed nodulation without altering root growth (Fig. 2a,b and Supplementary Fig. S4). By contrast, neither non-arabinosylated CLE-RS2 peptide (CLE-RS2p) nor Arabidopsis arabinosylated CLV3 peptide $\left(\left[\mathrm{Ara}_{3}\right] \mathrm{CLV} 3\right)$ showed any effect on nodulation (Fig. $2 \mathrm{a}-\mathrm{c}$ ), indicating that both the arabinose chain and the backbone peptide sequence are critical to the function of $\left[\mathrm{Ara}_{3}\right] \mathrm{CLE}-\mathrm{RS} 2$. We also confirmed that synthetic $\left[\mathrm{Ara}_{3}\right] \mathrm{CLE}-$ RS1 glycopeptide could suppress nodulation in a similar dosedependent manner as [Ara 3 CLE-RS2 (Fig. 2d). 

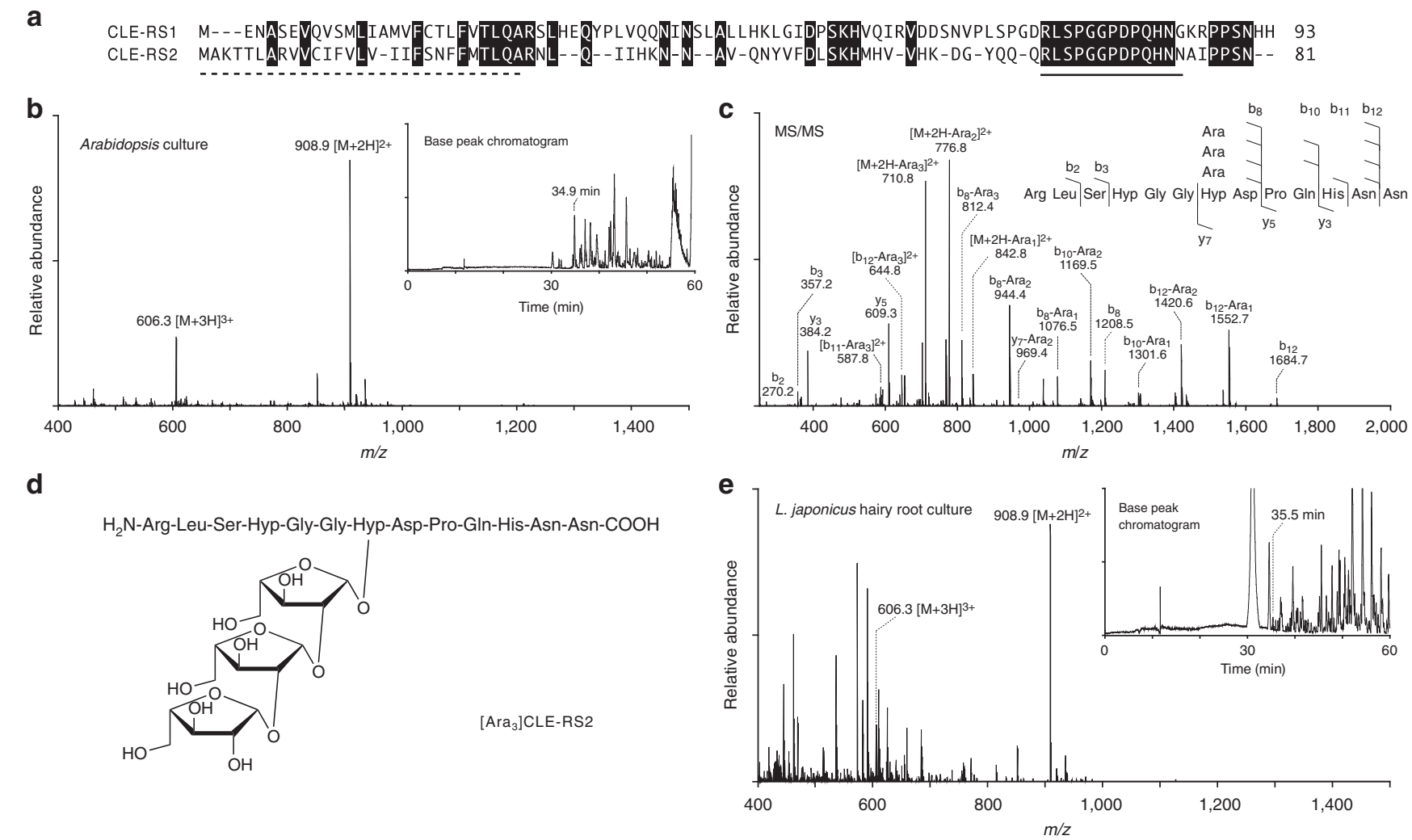

Figure 1 | Structural elucidation of mature CLE-RS2 peptide. (a) Amino-acid sequence alignment of the polypeptides encoded by CLE-RS1 and CLE-RS2. Mature $\left[\mathrm{Ara}_{3}\right] \mathrm{CLE}-\mathrm{RS} 2$ peptide-derived region is underlined, and the predicted secretion signal peptide is indicated by dashed line. The conserved region is shaded in black. (b) Detection of mature CLE-RS2 peptide secreted from Arabidopsis plants overexpressing CLE-RS2 at 34.9 min. Inset shows nanoLC-MS base peak chromatogram. (c) Nano-LC-MS/MS spectrum for the mature CLE-RS2 peptide. (d) Structure of the mature CLE-RS2 peptide ([Ara 3$]$ CLE-RS2). (e) Detection of mature CLE-RS2 peptide secreted by L. japonicus hairy roots overexpressing CLE-RS2 at 35.5 min. Inset shows nano-LCMS base peak chromatogram.

To confirm that the action of CLE-RS peptides is dependent on HAR1, we applied $\left[\mathrm{Ara}_{3}\right] \mathrm{CLE}-\mathrm{RS} 1$ and $\left[\mathrm{Ara}_{3}\right] \mathrm{CLE}-\mathrm{RS} 2$ to har1-4 mutant plants. The number of nodules on har1-4 mutants was not affected by application of [Ara 3 CLE-RS peptides even at $10 \mu \mathrm{M}$ (Fig. 2e). These results indicate that $\left[\mathrm{Ara}_{3}\right] \mathrm{CLE}-\mathrm{RS}$ peptides regulate nodule formation via HAR1.

[Ara ${ }_{3}$ CLE-RS glycopeptides directly bind to HAR1. Next, we examined the interaction of $\left[\mathrm{Ara}_{3}\right] \mathrm{CLE}-\mathrm{RS}$ peptides with HAR1 using $\left[\mathrm{Ara}_{3}\right] \mathrm{CLE}-\mathrm{RS} 2$ derivative tagged with ${ }^{125}$ I-labelled photoactivatable 4-azidosalicylic acid ([ $\left.\left.{ }^{125} \mathrm{I}\right] \mathrm{ASA}-\left[\mathrm{Ara}_{3}\right] \mathrm{CLE}-\mathrm{RS} 2\right)$ (Supplementary Fig. S5). Upon treatment with ultraviolet light, the azide group photolyses to form a reactive nitrene, which can form a covalent bond with receptor proteins. Microsomal fractions from tobacco BY-2 cells overexpressing HAR1-HaloTag (HAR1-HT) were photoaffinity labelled with $\left.{ }^{125}{ }^{12}\right] A S A-\left[\mathrm{Ara}_{3}\right]-$ CLE-RS2; after SDS-polyacrylamide gel electrophoresis and autoradiography, these fractions showed labelling of a $130-\mathrm{kD}$ band (Fig. 3a,b). This band disappeared completely after competitive inhibition by a 300 -fold excess of unlabelled $\left[\mathrm{Ara}_{3}\right] \mathrm{CLE}$ RS2, indicating that $\left[\mathrm{Ara}_{3}\right] \mathrm{CLE}-\mathrm{RS} 2$ specifically binds to HAR1. Notably, neither unglycosylated CLE-RS2p nor $\left[\mathrm{Ara}_{3}\right]$ CLV3 interfered with this binding (Fig. 3b,d), indicating that the arabinose chain and the amino acid sequence are critical for [Ara ${ }_{3}$ ]CLE-RS2 binding to HAR1. This absolute requirement for arabinose chain and sequence specificity in the receptor interaction explains the results of our nodulation bioassay. $\left[\mathrm{Ara}_{3}\right] \mathrm{CLE}$ RS1 also effectively competed with [125I]ASA-[ $\left.\mathrm{Ara}_{3}\right]$ CLE-RS2 for HAR1 binding in a similar affinity to that of $\left[\mathrm{Ara}_{3}\right] \mathrm{CLE}-\mathrm{RS} 2$, confirming that $\left[\mathrm{Ara}_{3}\right]$ CLE-RS1 also interacts with HAR1 (Fig. 3c).

We tested whether $\left[\mathrm{Ara}_{3}\right] \mathrm{CLE}-\mathrm{RS} 2$ could interact with a mutant receptor protein carrying the har1-4 mutation (L246F) ${ }^{9}$. Our analysis showed that $\left.{ }^{125} \mathrm{I}\right] \mathrm{ASA}-\left[\mathrm{Ara}_{3}\right] \mathrm{CLE}-\mathrm{RS} 2 \mathrm{did}$ not bind to the HAR $1^{\mathrm{L} 246 \mathrm{~F}}-\mathrm{HT}$ mutant receptor (Fig. 3a,b). Thus, loss of $\left[\mathrm{Ara}_{3}\right]$ CLE-RS2 responsiveness in the harl-4 mutant is reflected by a loss of $\left[\mathrm{Ara}_{3}\right] \mathrm{CLE}-\mathrm{RS} 2$ binding to HAR1. The har1-4 mutant carries a missense mutation in $\mathrm{Leu}^{246}$, which is located within LRR8 and which is known to have a critical role in ligand binding in the CLV1/BAM family receptor kinases in Arabidopsis ${ }^{28}$. We therefore conclude that both $\left[\mathrm{Ara}_{3}\right] \mathrm{CLE}-\mathrm{RS} 1$ and $\left[\mathrm{Ara}_{3}\right] \mathrm{CLE}-\mathrm{RS} 2$ can function as ligands for HAR1 receptor kinase.

[Ara 3 ]CLE-RS2 is transported through the xylem. As CLE-RS peptides are exclusively expressed in roots but act through HAR1 in the shoot ${ }^{8,9,16}$, the results of our ligand binding assay suggested a model in which $\left[\mathrm{Ara}_{3}\right]$ CLE-RS peptides are translocated from the root to the shoot where they bind to HAR1. As the xylem often mediates the transport of molecules from roots to shoots in higher plants ${ }^{29}$, we hypothesized that [Ara 3 CLE-RS peptides might be translocated via the xylem. Because efficient xylem sap collection from $L$. japonicus is technically difficult, we used the hairy root system in soybean plants that express $L$. japonicus CLE-RS2 following Agrobacterium-mediated transformation. This transformation system leads to the generation of chimeric plants that have transgenic hairy root systems attached to nontransformed stem and leaves. The shoots of 40-day-old plants were cut at the second internode above the hypocotyl, and xylem 


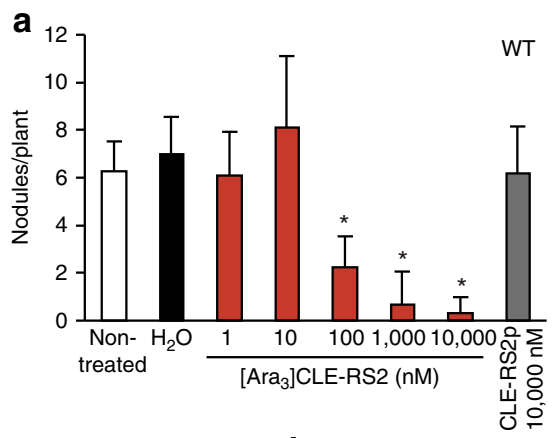

C

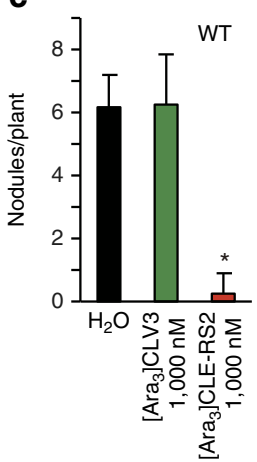

d
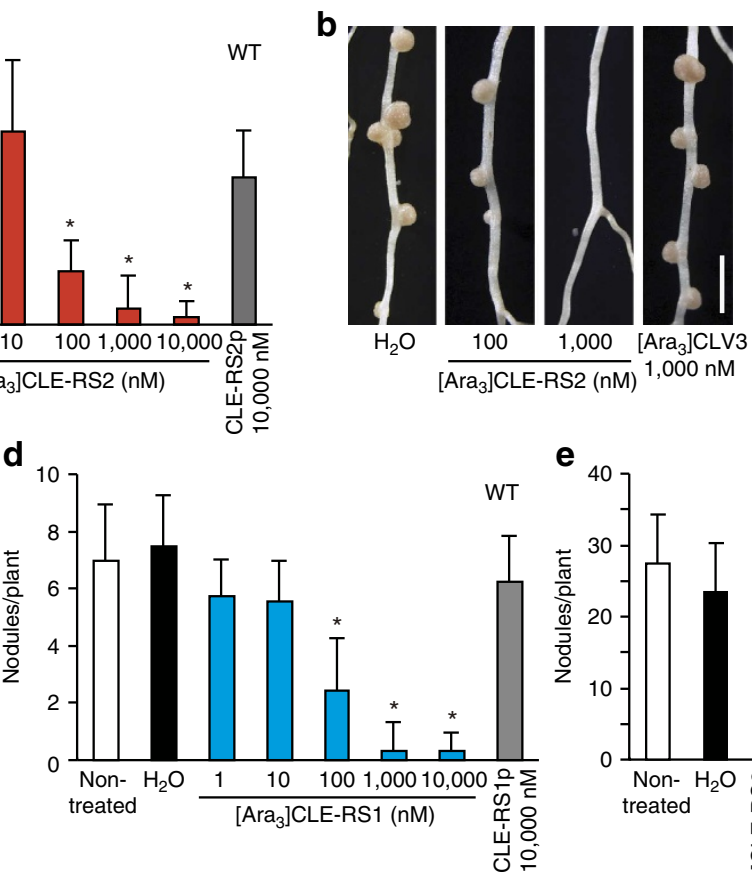

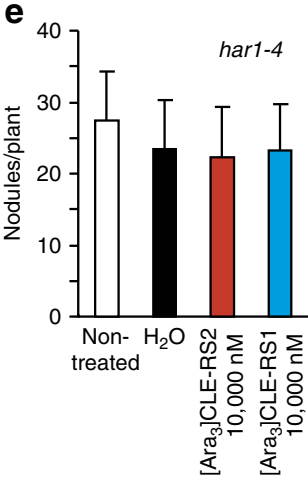

Figure 2 | Synthetic [Ara ${ }_{3}$ CLE-RS peptides suppress nodulation. (a) Effect of synthetic [Ara 3 ]CLE-RS2 on nodule formation in wild-type (WT) plants $(n=8-14)$. [Ara 3 ]CLE-RS2 was applied to the plants via a cut in the surface of the cotyledon. The number of nodules was counted 15 days after peptide application. (b) Nodules formed in roots of wild-type plants with or without application of $\left[\mathrm{Ara}_{3}\right] \mathrm{CLE}-\mathrm{RS} 2$ or $\left[\mathrm{Ara}_{3}\right] \mathrm{CLV} 3$. Photographs were taken 14 days after inoculation with rhizobia. Scale bars, $2 \mathrm{~mm}$. (c) Effect of $\left[\mathrm{Ara}_{3}\right] \mathrm{CLV} 3$ peptide on nodule formation in wild-type plants $(n=12)$. (d) Effect of synthetic $\left[\mathrm{Ara}_{3}\right] \mathrm{CLE}-\mathrm{RS} 1$ on nodule formation in wild-type plants $(n=8-14)$. (e) Effect of [Ara 3 CLE-RS2 and [Ara 3 CLE-RS1 on nodule formation in har7-4 mutant plants $(n=9-16)$. (a,c-e) Error bar indicates mean \pm s.d. Statistical differences were evaluated using a $t$-test. Statistically significant differences $(P<0.01)$ compared with $\mathrm{H}_{2} \mathrm{O}$-applied plants are indicated by asterisk.

a

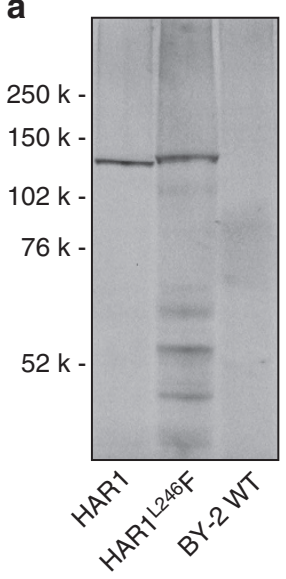

b

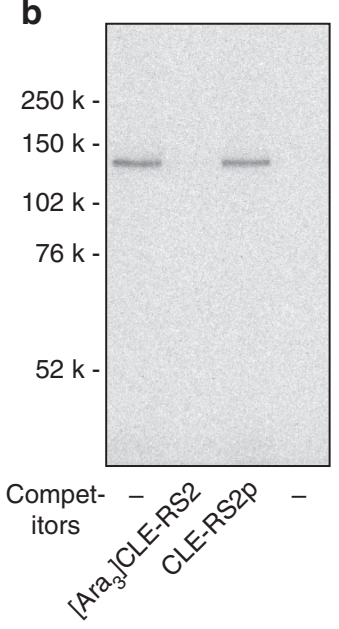

C

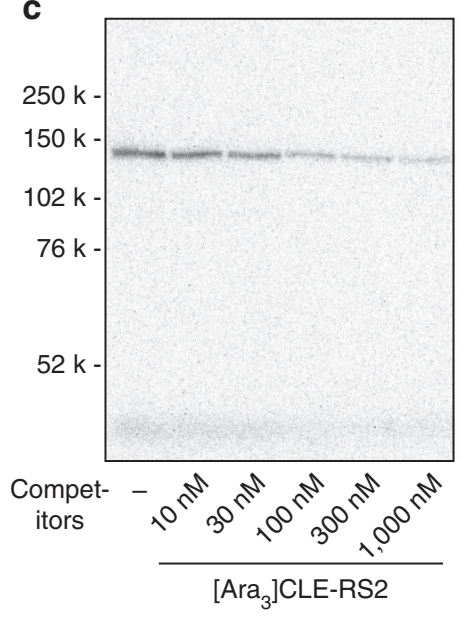

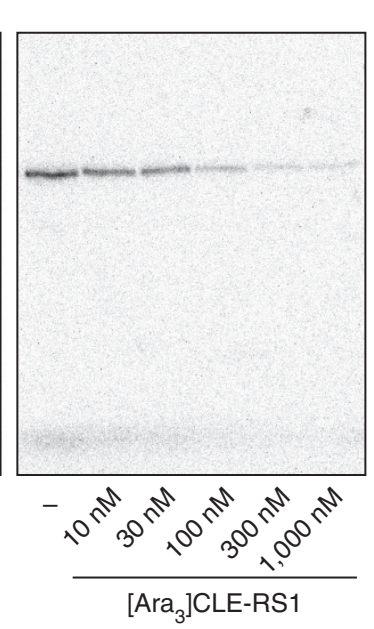

d

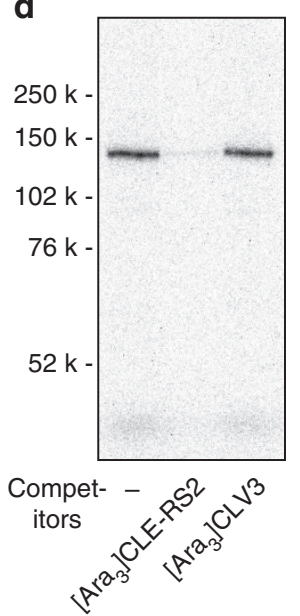

HAR1 HAR1L246F

Figure 3 | [ Ara $_{3}$ ]CLE-RS peptides directly bind to HAR1. (a) Expression of HAR1-HT and HAR1 ${ }^{\mathrm{L} 246 \mathrm{~F}}-\mathrm{HT}$ in transgenic BY-2 cells visualized by specific incorporation of a HaloTag TMR fluorescent dye. HaloTag protein is specifically labelled by HaloTag TMR that contains a haloalkane group. (b) Photoaffinity labelling of HAR1-HT and HAR1 ${ }^{\mathrm{L} 246 \mathrm{~F}}-\mathrm{HT}$ with $10 \mathrm{nM}\left[^{125} \mathrm{I}\right] \mathrm{ASA}-\left[\mathrm{Ara}_{3}\right] \mathrm{CLE}-\mathrm{RS} 2$ in the absence or presence of $300-\mathrm{foll}_{\mathrm{d}}$ excess of unlabelled $\left[\mathrm{Ara}_{3}\right]$ CLE-RS2 or CLE-RS2p. (c) Competitive displacement of [ $\left.{ }^{125} \mathrm{I}\right] \mathrm{ASA}-\left[\mathrm{Ara}_{3}\right] \mathrm{CLE}-\mathrm{RS} 2$ binding to HAR1-HT by various concentrations of unlabelled $\left[\mathrm{Ara}_{3}\right] \mathrm{CLE}-\mathrm{RS} 2$ and $\left[\mathrm{Ara}_{3}\right] \mathrm{CLE}-\mathrm{RS} 1$. (d) A 300-fold excess of unlabelled [Ara 3 ]CLV3 showed no competitive displacement of $\left[{ }^{125} \mathrm{I}_{\mathrm{I}} \mathrm{ASA}-\right.$ $\left[\mathrm{Ara}_{3}\right] \mathrm{CLE}-\mathrm{RS} 2$ binding to HAR1-HT.

sap was then collected for $10 \mathrm{~h}$. The sap was concentrated, partially purified and analysed by nano-LC-MS followed by nanoLC-MS/MS. The selected ion chromatogram for $m / z$ 908.9, which corresponds to $[\mathrm{M}+2 \mathrm{H}]^{2+}$, showed a peak at $34.8 \mathrm{~min}$ for xylem sap derived from plants with transgenic hairy roots overexpressing CLE-RS2 (Fig. 4a). An MS/MS analysis confirmed that the fragmentation pattern of this peak was identical to that of authentic $\left[\mathrm{Ara}_{3}\right]$ CLE-RS2 (Fig. 4c, see also Fig. 1c). This peak was 
not detected in xylem sap derived from plants with hairy roots transformed with an empty vector (Fig. 4b).

To eliminate the possibility that $\left[\mathrm{Ara}_{3}\right] \mathrm{CLE}-\mathrm{RS} 2$ in the xylem sap is due to unnatural leakage from tissues following its overexpression, we tested whether $\left[\mathrm{Ara}_{3}\right]$ CLE-RS2 could be detected in xylem sap when CLE-RS2 was driven by its own promoter. We transfected a genomic fragment of CLE-RS2 containing $3.2 \mathrm{~kb}$ upstream and $1.2 \mathrm{~kb}$ downstream regions from the open reading frame using the soybean hairy root system. The plants were inoculated with rhizobia, and 3 days after inoculation xylem sap was collected from the shoots. Partially purified xylem sap was directly analysed by nano-LC-MS. We confirmed that the selected ion chromatogram for $\mathrm{m} / \mathrm{z} 606.3$, which corresponds to $[\mathrm{M}+3 \mathrm{H}]^{3+}$ of $\left[\mathrm{Ara}_{3}\right]$ CLE-RS2, showed a detectable peak at 35.8 min (Fig. 4d). Thus, $\left[\mathrm{Ara}_{3}\right]$ CLE-RS2 was loaded into xylem sap under native expression levels. On the other hand, mass peaks corresponding to possible endogenous soybean CLE peptides were not detected in the partially purified xylem sap. Peptide recovery rate from xylem sap by $o$-chlorophenol extraction and peptide ionization efficiency by mass spectrometry are often affected by their amino-acid sequences ${ }^{24}$. Soybean CLE peptides, similar in structure to $L$. japonicus CLE-RS2 but with several amino-acid substitutions, might have lost in these steps. In conclusion, these results indicate that root-derived $\left[\mathrm{Ara}_{3}\right] \mathrm{CLE}-$ RS2 is transported from the root to the shoot through the xylem.

\section{Discussion}

Although the existence of a root-derived signal (also known as 'Q') was first proposed more than 20 years ago, it has long escaped identification ${ }^{5}$. A possible involvement of secreted peptides in autoregulation of nodulation was first suggested by identification of CLV1-like receptor kinase HAR1, and subsequently supported by identification of root-expressed CLE$R S$ peptide genes in L. japonicus that act upstream of HAR1. In contrast to the other known Arabidopsis CLE peptides, however, commercially available synthetic CLE domain peptides of CLE-RS family showed no biological activities ${ }^{16}$. Thus, until now, it was not clear whether CLE-RS peptides indeed act as ascending mobile signals that directly interact with HAR1. In this paper, we determined that the mature form of CLE-RS2 is a posttranslationally arabinosylated 13-amino-acid glycopeptide derived from the conserved C-terminal CLE domain. Arabinosylated CLE-RS peptides directly bound to HAR1 receptor kinase and showed strong biological activity to suppress nodulation at nanomolar concentrations when applied to shoots. In addition, $\left[\mathrm{Ara}_{3}\right] \mathrm{CLE}-\mathrm{RS} 2$ glycopeptide specifically expressed in the root can be detected in xylem sap collected from the shoot. Overall, our findings strongly suggest that arabinosylated CLE-RS peptides are the long sought rootderived signals involved in autoregulation of nodulation.

Our analyses showed that arabinosylation of CLE-RS1 and CLE-RS2 is critical for HAR1 binding and activation. This finding further supports the physiological importance of Hyp-arabinosylation in peptide signalling. Initial studies in Arabidopsis reported that arabinosylation of CLV3 glycopeptide considerably enhances its binding to CLV1 receptor kinase ${ }^{26}$. A recent conformational analysis of $\left[\mathrm{Ara}_{3}\right] \mathrm{CLV} 3$ showed that the arabinose chain of [Ara 3 ]CLV3 extends towards the C-terminal end of the peptide and causes distinct distortion in the C-terminal half of the peptide in a highly directional manner ${ }^{27}$. In this context, the arabinose chain of CLE-RS peptides is likely to have a role in maintaining a specific conformation of the peptide backbone rather than directly interacting with receptors. This speculation is supported by our observation that, despite the presence of the arabinose chain, $\left[\mathrm{Ara}_{3}\right] \mathrm{CLV} 3$ did not bind to HAR1.
Xylem primarily transports mineral-containing water from the soil to the aerial plant parts. However, we show here that the xylem also transports peptide signals that mediate systemic responses in plants. It remains to be elucidated whether the xylem loading of $\left[\mathrm{Ara}_{3}\right] \mathrm{CLE}-\mathrm{RS} 2$ glycopeptide is a passive (nonselective) or an active (selective) process; however, some known xylem proteins appear to be specifically expressed in roots suggesting the presence of a system for secretion into the xylem from the root cells ${ }^{30,31}$. How $\left[\mathrm{Ara}_{3}\right]$ CLE-RS2 glycopeptide is loaded into root xylem is a topic of great interest for future study.

The increased understanding of peptide signalling over the last two decades has established that secreted peptides principally have roles in local cell-to-cell communication within specific tissues in plant ${ }^{32}$. In this study, we provide the first indication that a secreted peptide ligand and a receptor kinase mediate longdistance organ-to-organ signalling that leads to a whole-plant systemic response. Although autoregulation of nodulation is a mechanism particularly associated with legumes, organ-to-organ communication is undoubtedly essential in all plant species for coordinated growth and for adaptation to environmental stresses at the whole-plant level $e^{33,34}$. Additionally, the fact that a large number of secreted peptide genes in the plant genome remain to be characterized, suggests that peptide signalling in plants might prove to be much more dynamic and diverse than previously anticipated.

\section{Methods}

Extraction of secreted peptides. Arabidopsis thaliana clv1-1 mutants were transformed using Agrobacterium tumefaciens GV3101, which harbours a p35S:CLE-RS2 construct ${ }^{16}$, via the floral dip method ${ }^{35}$. Transformants were selected using $20 \mu \mathrm{g} \mathrm{ml}^{-1}$ hygromycin. The resulting T3 plants (10 days after germination, DAG) were subjected to whole-plant submerged culture in $200 \mathrm{ml}$ medium $^{24}$. Secreted peptides accumulating after 24 days of culture were extracted by $o$-chlorophenol and fractionated in a gel-filtration column ${ }^{24,26}$.

Transformed hairy roots were induced by infecting L. japonicus B-129 Gifu with Agrobacterium rhizogenes AR1193 harbouring a $p 35 S$ :CLE-RS2 construct ${ }^{16}$. At 10-14 days after infection, green fluorescent protein-positive hairy roots were selected and cultured for a month in $100 \mathrm{ml}$ of B5 medium containing $1.0 \%$ sucrose; the cultures were maintained in the dark at $24^{\circ} \mathrm{C}$ with shaking at 100 r.p.m. The medium was then replaced with B5 medium containing $1.0 \%$ sucrose and $25 \mathrm{mM} \mathrm{CaCl}_{2}$, and the culture continued for 2 weeks without shaking. Secreted peptides that accumulated in the culture medium $(200 \mathrm{ml})$ were extracted and fractionated as above.

Nano-LC-MS and nano LC-MS/MS analyses. Nano-LC-MS analysis was performed using a DiNa-M splitless nano-HPLC system (KYA Technologies) connected to an LTQ Orbitrap XL mass spectrometer (Thermo Fisher Scientific). Aliquots of apoplastic small peptide fractions $(5-10 \mu \mathrm{l})$ were loaded onto a trap column ( $0.5 \mathrm{~mm}$ i.d. $\times 1 \mathrm{~mm}$ cartridge, C18; KYA Technologies) and washed with $0.1 \%$ formic acid. Peptides were subsequently eluted from the precolumn and separated on a long nano-column $(100 \mu \mathrm{m}$ i.d. $\times 750 \mathrm{~mm}$, MonoCap C18 High Resolution 750; GL Sciences), with a gradient of $2-50 \%$ acetonitrile (containing $0.1 \%$ formic acid) for $90 \mathrm{~min}$ at a flow rate of $500 \mathrm{nl} \mathrm{min}^{-1}$. The nano-HPLC eluate was introduced into a mass spectrometer via an electrospray ionization (ESI) interface at a spray voltage of $2.0 \mathrm{kV}$. The mass spectrometer was operated in positive ion mode with a capillary temperature of $160^{\circ} \mathrm{C}$. Mass spectra were obtained by scanning from $\mathrm{m} / z 400$ to $\mathrm{m} / z 1,500$. Nano-LC-MS/MS analysis was performed in manual scan mode, selecting the indicated molecular ion as the precursor ion at 30\% normalized collision energy using the higher energy collision dissociation mode.

Synthesis of [Ara ${ }_{3}$ ]CLE-RS peptides. $\mathrm{Asp}(t \mathrm{Bu})$-Pro-Gln(Trt)-His(Trt)Asn(Trt)-Asn(Trt)-resin was prepared by conventional solid-phase peptide synthesis using an ABI 431A peptide synthesizer. A mixture of Fmoc-[AcAra 3$]$ Hyp-OH $(6.0 \mathrm{mg}, 5.8 \mu \mathrm{mol})^{27}, 1$-hydroxybenzotriazole $(2.7 \mathrm{mg}$, $20 \mu \mathrm{mol}$ ), $O$-benzotriazole- $N, N, N^{\prime}, N^{\prime}$-tetramethyluronium hexafluorophosphate $(7.6 \mathrm{mg}, 20 \mu \mathrm{mol})$ and $N, N$-diisopropylethylamine $(7.0 \mu \mathrm{l}, 40 \mu \mathrm{mol})$ dissolved in dry $N$-methylpyrrolidone $(200 \mu \mathrm{l})$ was added to the peptide-resin $(25 \mu \mathrm{mol})$ preswollen with dry $N$-methylpyrrolidone $(50 \mu \mathrm{l})$. The mixture was stirred for $2 \mathrm{~h}$ at room temperature. Peptide-resin was recovered by filtration, and the remaining $\mathrm{N}$-terminal amino acids were added to the peptide using the peptide synthesizer. The synthesized peptide was deprotected and cleaved from the resin using trifluoroacetic acid/water $(95: 5 \mathrm{v} / \mathrm{v}, 1 \mathrm{ml})$ for $30 \mathrm{~min}$ and then precipitated using 

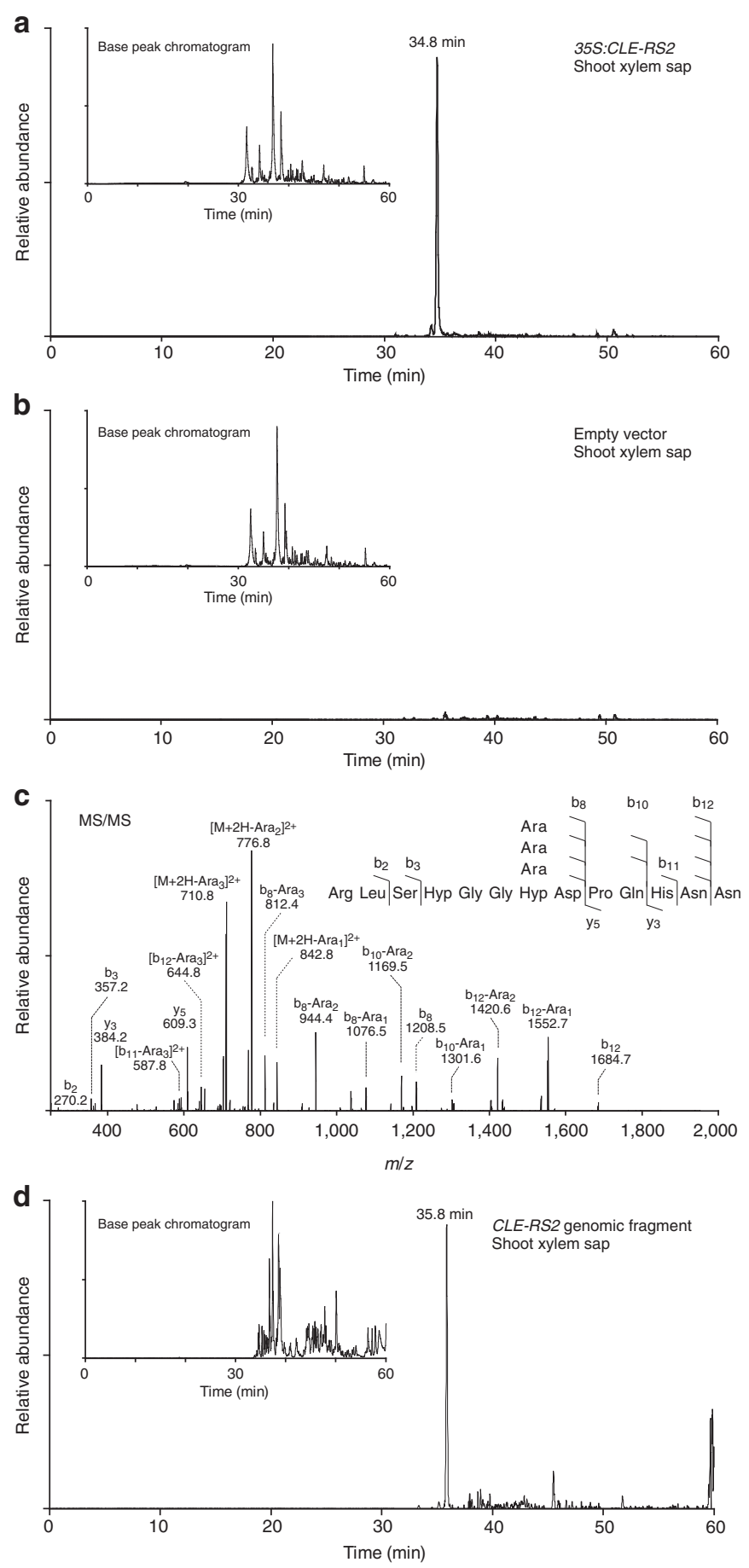

Figure 4 | Detection of root-derived [Ara 3 ]CLE-RS2 peptide in shoot xylem sap. (a) Nano-LC-MS chromatogram of shoot xylem sap collected from soybean plants, in which CLE-RS2 is overexpressed in transformed hairy roots. Selected ion chromatogram at $\mathrm{m} / \mathrm{z} 908.9$ that corresponds to $[\mathrm{M}+2 \mathrm{H}]^{2+}$ showed a peak at $34.8 \mathrm{~min}$. Inset shows the base peak chromatogram of shoot xylem sap. (b) Nano-LC-MS chromatogram of shoot xylem sap collected from soybean transformed with an empty vector. Selected ion chromatogram at $\mathrm{m} / \mathrm{z} 908.9$ indicates the absence of [Ara $\left.{ }_{3}\right]$ CLE-RS2. (c) MS/MS spectrum of the 34.8 min peak in a showed a fragmentation pattern identical to that of authentic [Ara 3 CLE-RS2. (d) Nano-LC-MS chromatogram of shoot xylem sap collected from soybean plants that developed transformed hairy roots after inoculation with a vector harbouring the CLE-RS2 genomic region. Selected ion chromatogram at $\mathrm{m} / \mathrm{z}$ 606.3 that corresponds to $[\mathrm{M}+3 \mathrm{H}]^{3+}$ showed a peak at $35.8 \mathrm{~min}$. Inset shows the base peak chromatogram of the xylem sap. ether $(10 \mathrm{ml})$ at $-20^{\circ} \mathrm{C}$ for $5 \mathrm{~min}$. The precipitated peptide was washed twice with cold ether, dissolved in water and lyophilized. Crude peptide was dissolved in dry methanol $(3 \mathrm{ml})$ and treated with sodium methoxide $(28 \%$ solution, $60 \mu \mathrm{l})$ at room temperature for $1 \mathrm{~h}$. The reaction was terminated by adding acetic acid $(60 \mu \mathrm{l})$. HPLC purification using an amide column (TSK-gel amide-80, TOSOH) gave analytically pure $\left[\mathrm{Ara}_{3}\right]$ CLE-RS2 $(1.5 \mathrm{mg})$. For the synthesis of [ $\left.\mathrm{Ara}_{3}\right] \mathrm{CLE}-\mathrm{RS} 1$ glycopeptide, $\mathrm{Asp}(t \mathrm{Bu})$-Pro-Gln(Trt)-His(Trt)-Asn(Trt)-Gly-resin was used as the starting material.

Bioassay. Sterilized seeds of L. japonicus B-129 Gifu (wild type) or the hypernodulating mutant harl-4 (sym78-1) were germinated and grown in plastic boxes with sterilized vermiculite moistened with liquid Broughton and Dilworth (B\&D) medium in a growth cabinet $\left(22^{\circ} \mathrm{C}, 16 \mathrm{~h}\right.$ light $/ 22{ }^{\circ} \mathrm{C}, 8 \mathrm{~h}$ dark). At $6 \mathrm{DAG}$, the distal half of each cotyledon was excised and the remaining half was inserted into a plastic tube filled with peptide solution. Next day, plants were inoculated with $M$ loti MAFF303099 and were grown for a further 14 days. The peptide solution was supplemented every 3 days to ensure continuous immersion of the cut cotyledon surface. Nodule numbers were counted at 14 days after inoculation. The effectiveness of the technique was confirmed using uptake of blue dye (Palace Chemicals, Japan) into primary shoot leaves.

Synthesis of photoactivatable ASA-[Ara 3 CLE-RS2. The Fmoc-protected $\left[\mathrm{Ara}_{3}\right] \mathrm{CLE}-\mathrm{RS} 2$ analogue, Fmoc- $\left[\mathrm{Lys}^{2}\right]\left[\mathrm{Ara}_{3}\right]$ CLE-RS2, was synthesized as described above. 4-Azidosalicylic acid succinimidyl ester $(0.2 \mathrm{mg}$, Pearce), Fmoc- $\left[\mathrm{Lys}^{2}\right]\left[\mathrm{Ara}_{3}\right]$ CLE-RS2 $(1.4 \mathrm{mg})$ and $\mathrm{NaHCO}_{3}(1.0 \mathrm{mg})$ were dissolved in $100 \mu \mathrm{l}$ of $50 \%$ acetonitrile and stirred for $2 \mathrm{~h}$ in the dark at room temperature. Crude peptide was purified by reverse-phase HPLC and lyophilized to yield Fmoc[(4-azidosalicyl)Lys $\left.{ }^{2}\right]\left[\mathrm{Ara}_{3}\right] \mathrm{CLE}-\mathrm{RS} 2 ; 50 \mu \mathrm{l}$ of $50 \%$ piperidine in acetonitrile was added to the purified peptide, followed by incubation for $1 \mathrm{~h}$ in the dark at room temperature. The deprotected peptide was further purified by reverse-phase HPLC and lyophilized to obtain analytically pure $\left[\left(4\right.\right.$-azidosalicyl) Lys $\left.{ }^{2}\right]\left[\mathrm{Ara}_{3}\right]$ CLE-RS2

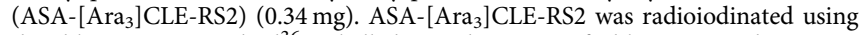
the chloramine $\mathrm{T}_{\text {method }}{ }^{36}$. Labelled peptide was purified by reverse-phase HPLC to yield analytically pure $\left[{ }^{125} \mathrm{I}\right] \mathrm{ASA}-\left[\mathrm{Ara}_{3}\right] \mathrm{CLE}-\mathrm{RS} 2$ with a specific radioactivity of $85 \mathrm{Ci} \mathrm{mmol}^{-1}$.

Expression of HAR1-HT and photoaffinity labelling. To overexpress HAR1-HT in tobacco BY-2 cells, we PCR amplified the genomic fragment of HAR1 corresponding to the $\mathrm{Met}^{1}$ to $\mathrm{Glu}^{702}$ region, and the cDNA fragment of HaloTag (Promega) containing the entire open reading frame of HaloTag. The two fragments were cloned in translational fusion by three-component ligation into the BamHI/SacI-digested binary vector pBI121 using an In-Fusion HD Cloning Kit (Clontech). To prepare HAR1 ${ }^{\text {L246F }}$-HT, the amino-acid substitution L246F was introduced into HAR1-HT by PCR-based site-directed mutagenesis. Transformation of tobacco BY-2 cells and preparation of microsomal fractions were described previously ${ }^{36}$. To confirm expression, HaloTag-fused proteins were specifically labelled using HaloTag TMR (Promega), separated by SDSpolyacrylamide gel electrophoresis, and visualized by a fluorescent image analyzer with a $523 \mathrm{~nm}$ excitation filter and a $580 \mathrm{~nm}$ emission filter. Photoaffinity labelling was performed as described ${ }^{28}$.

Hairy root transformation and collection of xylem sap. G. max Enrei seedlings (5 DAG) were inoculated with A. rhizogenes K599 harbouring either a CLE-RS2overexpressing construct or an empty vector. Hairy root transformation was induced $^{37}$, and the plants were grown on vermiculite. Three weeks after inoculation, untransformed roots were removed, and plants with green fluorescent protein-positive hairy roots were transplanted into fresh sterilized vermiculite. Three weeks after replanting, the stem was cut at the second internode above the hypocotyl and xylem sap was collected. Approximately $30 \mathrm{ml}$ xylem sap was obtained from 4 CLE-RS2-overexpressing plants, and almost same amount of xylem sap was obtained from five plants transformed with an empty vector. Peptides in the xylem sap were extracted, size-fractionated and subjected to nanoLC-MS and nano-LC-MS/MS analyses as above.

To express CLE-RS2 under the control of its own promoter, a genomic fragment of CLE-RS2 containing an approximately $3.2 \mathrm{~kb}$ upstream region and a $1.2 \mathrm{~kb}$ downstream region from the open reading frame was cloned using the primers $5^{\prime}$-AGGCTTTAACTCATTGCATC- ${ }^{\prime}$ and $5^{\prime}$-ATTGCACAGTAATGGTTTCC- ${ }^{\prime}$. Hairy root transformation was performed as above. Three weeks after replanting, the plants were inoculated with Bradirhizobium japonicum A1017; after 3 days, $1.5 \mathrm{ml}$ xylem sap was obtained from each plant. Peptides in the xylem sap were extracted by $o$-chlorophenol and subjected to nano-LC-MS and nano-LC-MS/MS analyses as above.

\section{References}

1. Caetano-Anolles, G. \& Gresshoff, P. M. Plant genetic control of nodulation. Annu. Rev. Microbiol. 45, 345-382 (1991).

2. Oka-Kira, E. \& Kawaguchi, M. Long-distance signaling to control root nodule number. Curr. Opin. Plant Biol. 9, 496-502 (2006). 
3. Magori, S. \& Kawaguchi, M. Long-distance control of nodulation: molecules and models. Mol. Cells 27, 129-134 (2009).

4. Ferguson, B. J. et al. Molecular analysis of legume nodule development and autoregulation. J. Integr. Plant Biol. 52, 61-76 (2010).

5. Caetano-Anolles, G. \& Gresshoff, P. M. Early induction of feedback regulatory responses governing nodulation in soybean. Plant Sci. 71, 69-81 (1990).

6. Kosslak, R. M. \& Bohlool, B. B. Suppression of nodule development of one side of a split-root system of soybeans caused by prior inoculation of the other side. Plant Physiol. 75, 125-130 (1984).

7. Olsson, J. E., Nakao, P., Bohlool, B. B. \& Gresshoff, P. M. Lack of systemic suppression of nodulation in split root systems of supernodulating soybean (Glycine max [L.] Merr.) mutants. Plant Physiol. 90, 1347-1352 (1989).

8. Krusell, L. et al. Shoot control of root development and nodulation is mediated by a receptor-like kinase. Nature 420, 422-426 (2002).

9. Nishimura, R. et al. HAR1 mediates systemic regulation of symbiotic organ development. Nature 420, 426-429 (2002).

10. Wopereis, J. et al. Short root mutant of Lotus japonicus with a dramatically altered symbiotic phenotype. Plant J. 23, 97-114 (2000).

11. Searle, I. R. et al. Long-distance signaling in nodulation directed by a CLAVATA1-like receptor kinase. Science 299, 109-112 (2003).

12. Schnabel, E., Journet, E. P., de Carvalho-Niebel, F., Duc, G. \& Frugoli, J. The Medicago truncatula SUNN gene encodes a CLV1-like leucine-rich repeat receptor kinase that regulates nodule number and root length. Plant Mol. Biol. 58, 809-822 (2005)

13. Clark, S. E., Williams, R. W. \& Meyerowitz, E. M. The CLAVATA1 gene encodes a putative receptor kinase that controls shoot and floral meristem size in Arabidopsis. Cell 89, 575-585 (1997).

14. Fletcher, J. C., Brand, U., Running, M. P., Simon, R. \& Meyerowitz, E. M. Signaling of cell fate decisions by CLAVATA3 in Arabidopsis shoot meristems. Science 283, 1911-1914 (1999).

15. Cock, J. M. \& McCormick, S. A large family of genes that share homology with CLAVATA3. Plant Physiol. 126, 939-942 (2001).

16. Okamoto, S. et al. Nod factor/nitrate-induced CLE genes that drive HAR1mediated systemic regulation of nodulation. Plant Cell Physiol. 50, 67-77 (2009).

17. Mortier, V., De Wever, E., Vuylsteke, M., Holsters, M. \& Goormachtig, S. Nodule numbers are governed by interaction between CLE peptides and cytokinin signaling. Plant J. 70, 367-376 (2012).

18. Mortier, V. et al. CLE peptides control Medicago truncatula nodulation locally and systemically. Plant Physiol. 153, 222-237 (2010).

19. Reid, D. E., Ferguson, B. J. \& Gresshoff, P. M. Inoculation- and nitrate-induced CLE peptides of soybean control NARK-dependent nodule formation. Mol. Plant Microbe Interact. 24, 606-618 (2011).

20. Mortier, V. et al. Search for nodulation-related CLE genes in the genome of Glycine max. J. Exp. Bot. 62, 2571-2583 (2011).

21. Lim, C. W., Lee, Y. W. \& Hwang, C. H. Soybean nodule-enhanced CLE peptides in roots act as signals in GmNARK-mediated nodulation suppression. Plant Cell Physiol. 52, 1613-1627 (2011).

22. Matsubayashi, Y. Post-translational modifications in secreted peptide hormones in plants. Plant Cell Physiol. 52, 5-13 (2011).

23. Gao, X. \& Guo, Y. CLE peptides in plants: proteolytic processing, structureactivity relationship, and ligand-receptor interaction. J. Integr. Plant Biol. 54, 738-745 (2012)

24. Ohyama, K., Ogawa, M. \& Matsubayashi, Y. Identification of a biologically active, small, secreted peptide in Arabidopsis by in silico gene screening, followed by LC-MS-based structure analysis. Plant J. 55, 152-160 (2008).

25. Amano, Y., Tsubouchi, H., Shinohara, H., Ogawa, M. \& Matsubayashi, Y. Tyrosine-sulfated glycopeptide involved in cellular proliferation and expansion in Arabidopsis. Proc. Natl Acad. Sci. USA 104, 18333-18338 (2007).

26. Ohyama, K., Shinohara, H., Ogawa-Ohnishi, M. \& Matsubayashi, Y. A glycopeptide regulating stem cell fate in Arabidopsis thaliana. Nat. Chem. Biol. 5, 578-580 (2009).
27. Shinohara, H. \& Matsubayashi, Y. Chemical synthesis of Arabidopsis CLV3 glycopeptide reveals the impact of hydroxyproline arabinosylation on peptide conformation and activity. Plant Cell Physiol. 54, 369-374 (2013).

28. Shinohara, H., Moriyama, Y., Ohyama, K. \& Matsubayashi, Y. Biochemical mapping of a ligand-binding domain within Arabidopsis BAM1 reveals diversified ligand recognition mechanisms of plant LRR-RKs. Plant J. 70, 845-854 (2012).

29. Schachtman, D. P. \& Goodger, J. Q. Chemical root to shoot signaling under drought. Trends Plant Sci. 13, 281-287 (2008).

30. Masuda, S., Kamada, H. \& Satoh, S. Chitinase in cucumber xylem sap. Biosci. Biotechnol. Biochem. 65, 1883-1885 (2001).

31. Masuda, S., Sakuta, C. \& Satoh, S. cDNA cloning of a novel lectin-like xylem sap protein and its root-specific expression in cucumber. Plant Cell Physiol. 40, 1177-1181 (1999).

32. Matsubayashi, Y. Small post-translationally modified peptide signals in Arabidopsis. Arabidopsis Book 9, e0150 (2011).

33. Turnbull, C. Long-distance regulation of flowering time. J. Exp. Bot. 62, 4399-4413 (2011).

34. Shah, J. \& Zeier, J. Long-distance communication and signal amplification in systemic acquired resistance. Front Plant Sci. 4, 30 (2013).

35. Clough, S. J. \& Bent, A. F. Floral dip: a simplified method for Agrobacterium mediated transformation of Arabidopsis thaliana. Plant J. 16, 735-743 (1998)

36. Ogawa, M., Shinohara, H., Sakagami, Y. \& Matsubayashi, Y. Arabidopsis CLV3 peptide directly binds CLV1 ectodomain. Science 319, 294 (2008).

37. Kouchi, H., Takane, K., So, R. B., Ladha, J. K. \& Reddy, P. M. Rice ENOD40: isolation and expression analysis in rice and transgenic soybean root nodules. Plant J. 18, 121-129 (1999).

\section{Acknowledgements}

We thank Hiroshi Kouch for providing A. rhizogenes K599, Mari Kanaoka-Banba and Hiroko Yamaya for helpful advice on the soybean hairy transformation and culture protocols, Mari Ogawa-Ohnishi for site-directed mutagenesis of HAR1-HT, Sachiko Tanaka, Michiko Ichikawa and Yuko Ogawa for supporting to prepare soybean culture. We would also like to thank NIBB Model Plant Research Facility for providing facilities for the culture of Arabidopsis and soybeans and the Radioisotope Center for support in the radioisotope experiments. This research was supported by Grant-in-Aid for JSPS Research Fellows from the Japan Society for the Promotion of Science (JSPS; no. A2406127 to S.O.), Funding Program for Next Generation World-Leading Researchers from JSPS (no. GS025 to Y.M.), Grant-in-Aid for Scientific Research for Priority Areas from the Ministry of Education, Culture, Sports, Science, and Technology (MEXT; no. 19060010 to Y.M. and no. 23012020 to H.S.), and Grants-in-Aid for Scientific Research from the MEXT (no. 22128006 to M.K. and no. 25221105 to Y.M.)

\section{Author contributions}

S.O., T.M. and Y.M. performed the MS analyses, Y.M. synthesized the glycopeptides, H.S conducted the binding assay and S.O. performed all the other experiments. S.O., Y.M. and M.K. designed research, analysed data and wrote the paper.

\section{Additional information}

Supplementary Information accompanies this paper at http://www.nature.com/ naturecommunications

Competing financial interests: The authors declare no competing financial interests.

Reprints and permission information is available online at http://npg.nature.com/ reprintsandpermissions/

How to cite this article: Okamoto, S. et al. Root-derived CLE glycopeptides control nodulation by direct binding to HAR1 receptor kinase. Nat. Commun. 4:2191 doi: 10.1038/ncomms3191 (2013). 